\title{
A GENERAL ALGORITHM FOR COMPRESSIBLE AND INCOMPRESSIBLE FLOWS. PART III: THE SEMI-IMPLICIT FORM
}

\author{
R. CODINA ${ }^{1 *}$, M. VÁZQUEZ ${ }^{1}$ AND O. C. ZIENKIEWICZ ${ }^{2}$ \\ ${ }^{1}$ International Center for Numerical Methods in Engineering, Universitat Politècnica de Catalunya, Gran Capità s/n, \\ Edifici C1, E-08034 Barcelona, Spain \\ ${ }^{2}$ Institute of Numerical Methods in Engineering, University of College of Swansea (UCS), Swansea SA2 8PP, U.K.
}

\begin{abstract}
SUMMARY
In this paper we consider some particular aspects related to the semi-implicit version of a fractional step finite element method for compressible flows that we have developed recently. The first is the imposition of boundary conditions. We show that no boundary conditions at all need to be imposed in the first step where an intermediate momentum is computed. This allows us to impose the real boundary conditions for the pressure, a point that turns out to be very important for compressible flows.

The main difficulty of the semi-implicit form of the scheme arises in the solution of the continuity equation, since it involves both the density and the pressure. These two variables can be related through the equation of state, which in turn introduces the temperature as a variable in many cases. We discuss here the choice of variables (pressure or density) and some strategies to solve the continuity equation.

The final point that we study is the behaviour of the scheme in the incompressible limit. It is shown that the method has an inherent pressure dissipation that allows us to reach this limit without having to satisfy the classical compatibility conditions for the interpolation of the velocity and the pressure. (C) 1998 John Wiley \& Sons, Ltd.
\end{abstract}

Int. J. Numer. Meth. Fluids, 27: 13-32 (1998)

KEY WORDS: splitting; pressure stabilization; characteristic schemes

\section{INTRODUCTION}

Although the use of fractional step methods has been widespread for incompressible flow problems since the original work of Chorin ${ }^{1}$ and Temam, ${ }^{2}$ less attention has been paid to the development of schemes of this type for high-speed compressible flows. Recently we have developed one such methods $^{3,4}$ using the finite element method for the spatial discretization (see Reference 5 for another algorithm using a non-conservation form of the flow equations). In this paper we address some particular aspects related to the semi-implicit form of the scheme.

\footnotetext{
* Correspondence to: R. Codina, International Centre for Numerical Methods in Engineering, Universitat Politècnica de Catalunya, Gran Capità s/n, Edifici C1, E-08034 Barcelona, Spain
}

Contract grant sponsor: NASA; Contract grant number: NAGW/2127, Ames Control Number 90-144 
The first point discussed is the imposition of boundary conditions, which is always controversial in the application of fractional step methods. In the first step of our scheme we compute an intermediate momentum for which no boundary conditions need to be imposed; that is to say, this intermediate momentum is computed also on the boundary. For viscous flows this leads to the computation of a boundary integral involving the viscous stresses. This allows us to impose the real boundary conditions for the pressure. The rest of the boundary conditions can be specified in the usual manner.

The main difficulty in the implementation of the algorithm arises in the solution of the continuity equation, since it involves both the density and the pressure. These two variables can be related through the equation of state, and one of the most common cases for compressible flows being that corresponding to perfect gases. However, this equation introduces a new variable, namely the temperature, and therefore the final system of equations to be solved at each time step must be solved iteratively. We describe several possibilities and discuss their performance. In particular, it is possible to obtain the temperature explicitly from the energy equation written in non-conservation form and to use it in the continuity equation. We have found in several numerical experiments that using this version of the energy equation rather than the conservative one may yield shocks placed at a wrong position and with a wrong strength, but the procedure works well if the flow has no shocks. Another possibility is to use a guess for the temperature and to correct it at the end of the step using the total energy as unknown and thus the energy equation in conservation form. We present a classical benchmark problem using this approach.

Another aspect of the scheme that we study is its application in the incompressible limit. It is

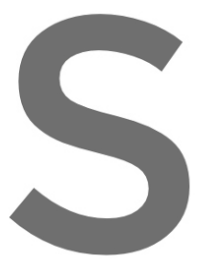
shown that the method allows us to reach this limit without having to satisfy the classical compatibility conditions for the interpolation of the velocity and the pressure. This is so owing to an inherent pressure dissip operators computed in explaining in part why incompressible limit wi
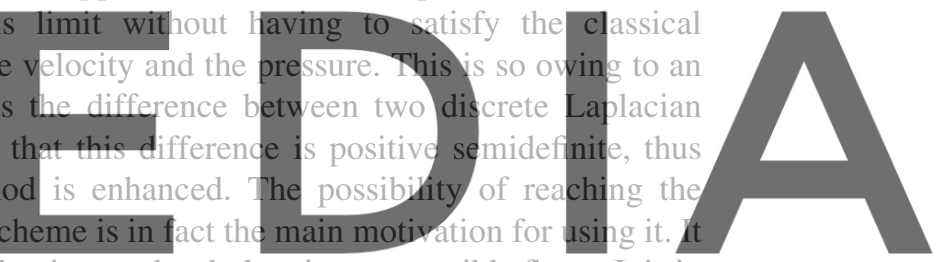

has been employed for example in Reference 6 for laminar and turbulent incompressible flows. It is in this sense that the scheme can be termed 'general'. The stabilization properties of fractional step

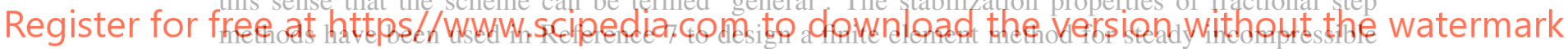
flows that allows the use of equal velocity-pressure interpolations. Other ways of achieving this are extensions to the compressible case of methods designed for incompressible flows. A first possibility is the use of mixed interpolations satisfying the classical inf-sup stability condition. The use of such interpolations also for compressible flows has been advocated for example in References 8-10. The need for interpolations satisfying also a certain inf-sup condition in a simple case of compressible flows is analysed in Reference 11. Another possibility is to extend stabilization techniques known to work well for incompressible flows with equal interpolation for all the variables to the compressible case. This has been used for example in References 12 and 13, where the Galerkin/least squares method is applied to compressible flows.

The paper is organized as follows. In Section 2 we describe briefly our basic split algorithm introduced in Reference 3. The imposition of boundary conditions is addressed in Section 3, where the weak form of the problem is established. In Section 4 we consider particular flows and solution strategies for them using the present algorithm, all this at the discrete level; we start with incompressible and slightly compressible flows, then continue with barotropic flows and finally with the most complex case of perfect gases. In all cases the objective is to write the particular expression of the continuity equation according to the type of flow, choosing either the pressure or the density as variable. In Section 5 we present the numerical results obtained for classical benchmark problems in three different types of flow regimes, namely fully incompressible, barotropic and supersonic perfect gas. Finally, some conclusions are drawn. 


\section{FRACTIONAL STEP METHOD FOR COMPRESSIBLE FLOWS}

In this section we describe briefly the fractional step method presented in References 3 and 4. Let us write the compressible Navier-Stokes equations in conservation form in a Cartesian co-ordinate system $\left(x_{1}, x_{2}, x_{3}\right)$ as

$$
\frac{\partial \mathbf{V}}{\partial t}+\frac{\partial \mathbf{F}_{i}}{\partial x_{i}}+\frac{\partial \mathbf{G}_{i}}{\partial x_{i}}+\mathbf{Q}=\mathbf{0}
$$

where, in the $3 \mathrm{D}$ case,

$$
\mathbf{V}=\left[\begin{array}{c}
\rho \\
\rho u_{1} \\
\rho u_{2} \\
\rho u_{3} \\
\rho e
\end{array}\right], \quad \mathbf{F}_{i}=\left[\begin{array}{c}
\rho u_{i} \\
\rho u_{1} u_{i}+\delta_{1 i} p \\
\rho u_{2} u_{i}+\delta_{2 i} p \\
\rho u_{3} u_{i}+\delta_{3 i} p \\
u_{i}(\rho e+p)
\end{array}\right], \quad \mathbf{G}_{i}=\left[\begin{array}{c}
0 \\
-\tau_{1 i} \\
-\tau_{2 i} \\
-\tau_{3 i} \\
-\left(k \partial T / \partial x_{i}\right)-\tau_{i j} u_{j}
\end{array}\right], \quad \mathbf{Q}=\left[\begin{array}{c}
0 \\
\rho g_{1} \\
\rho g_{2} \\
\rho g_{3} \\
\rho\left(g_{i} u_{i}+r\right)
\end{array}\right],
$$

\section{with}

$$
\tau_{i j}=\mu\left(\frac{\partial u_{i}}{\partial x_{j}}+\frac{\partial u_{j}}{\partial x_{i}}-\frac{2}{3} \frac{\partial u_{k}}{\partial x_{k}} \delta_{i j}\right) .
$$

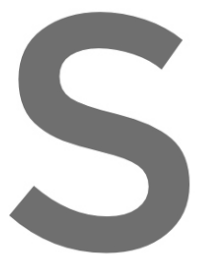

Here $\rho$ is the density, $u_{i}$ the pressure, $T$ is the th source, $k$ is the therm be supplied with an related to $T$ and $u_{i}$ thro volume. Here and belov
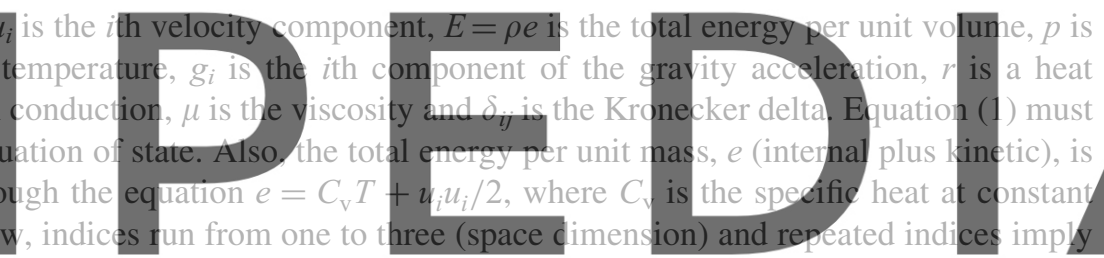
summation.

Let us write the conservation equations for the momentum $U_{i}=\rho u_{i}$ and the density $\rho$ (continuity

Register for frequatiat hatstps//wwW.scipedia.com to download the version without the watermark

$$
\begin{aligned}
& \frac{\partial U_{i}}{\partial t}=M_{i}-\frac{\partial p}{\partial x_{i}}=: R_{i}, \\
& \frac{\partial \rho}{\partial t}=-\frac{\partial U_{i}}{\partial x_{i}},
\end{aligned}
$$

where $R_{i}$ is the $i$ th component of the steady state residual and we have used the abbreviation

$$
M_{i}:=-\frac{\partial}{\partial x_{j}}\left(\rho u_{i} u_{j}-\tau_{i j}\right)-\rho g_{i} .
$$

The convective contribution $u_{j} \partial\left(\rho u_{i}\right) / \partial x_{j}$ appearing in $M_{i}$ could lead to numerical instabilities if the standard Galerkin formulation is used to discretize the space. In order to stabilize this effect, we first discretize equation (4) in time along the characteristics of the total derivative $\partial / \partial t+u_{j} \partial / \partial x_{j}$ as explained in Reference 3 . This leads to the equations

$$
\begin{aligned}
& \frac{\Delta U_{i}^{n}}{\Delta t}=M_{i}^{n}-\frac{\partial p^{n+\theta_{2}}}{\partial x_{i}}-\frac{\Delta t}{2} u_{k}^{n} \frac{\partial R_{i}^{n}}{\partial x_{k}}, \\
& \frac{\Delta \rho^{n}}{\Delta t}=-\frac{\partial U_{i}^{n+\theta_{1}}}{\partial x_{i}},
\end{aligned}
$$


where $\Delta t$ is the time step size (assumed to be constant for simplicity), the superscripts denote the time step level, $\theta_{1}, \theta_{2} \in[0,1]$ and we use the notation $f^{n+\theta}=\theta f^{n+1}+(1-\theta) f^{n}$ and $\Delta f^{n}=f^{n+1}-f^{n}$ for any function $f$ and $\theta \in[0,1]$. Observe that in (7) all the terms except the pressure gradient are treated explicitly. This simplifies the exposition for the following splitting method, even though the continuous problem is not well posed if there are boundary conditions of Dirichlet type for the velocity (or the momentum). The use of this scheme can be justified by assuming that the viscous term is first treated implicitly, i.e. $\Delta M_{i}:=-\left(\partial / \partial x_{j}\right)\left(\tau_{i j}^{n+1}-\tau_{i j}^{n}\right)$ is added to the RHS of (7), and then the contribution of $\Delta M_{i}$ is neglected in the discrete problem.

Let

$$
\Delta \tilde{U}_{i}^{n}:=\Delta U_{i}^{n}+\Delta t \frac{\partial p^{n+\theta_{2}}}{\partial x_{i}} .
$$

Having introduced this new variable, equations (7) and (8) can be written as

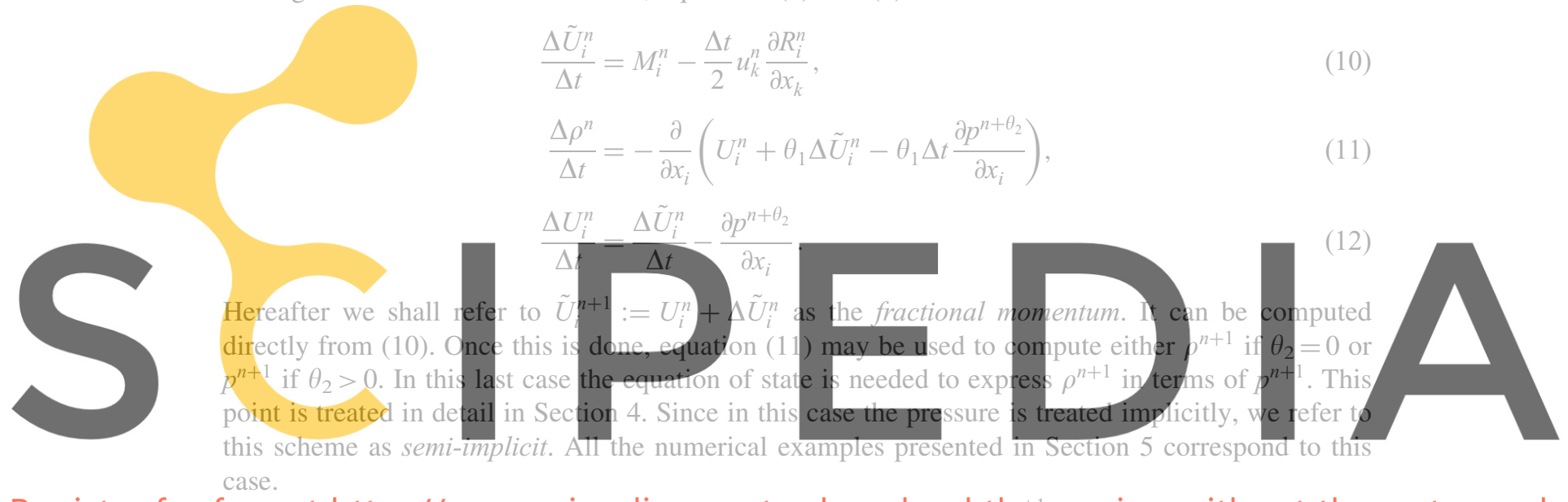

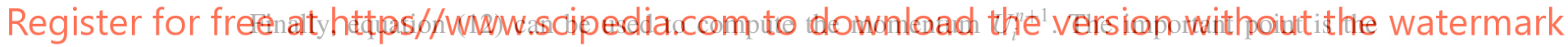
substitution of $\Delta U_{i}^{n}$ in (11) using equation (9), all this at the continuous level. This will lead to a stabilizing pressure dissipation term in the discrete finite element scheme that allows us to use this scheme for incompressible flows with the same velocity-pressure finite element interpolation if the semi-implicit version of the algorithm is employed.

\section{BOUNDARY CONDITIONS AND WEAK FORM}

\subsection{Fractional momentum equation}

Let us now obtain the weak form of (10)-(12). Considering first equation (10), let $\tilde{W}_{i}$ be the $i$ th component of the test function for the fractional momentum. We shall compute it in the problem domain $\Omega$ and also on its boundary $\Gamma=\partial \Omega$ and therefore $\tilde{W}_{i}$ is subject to no conditions. Multiplying equation (10) by $\tilde{W}_{i}$, integrating over $\Omega$ and integrating the viscous term and the term coming from the discretization along the characteristics by parts, we get

$$
\begin{aligned}
\int_{\Omega} \tilde{W}_{i} \frac{\Delta \tilde{U}_{i}^{n}}{\Delta t} \mathrm{~d} \Omega= & -\int_{\Omega} \tilde{W}_{i}\left(\frac{\partial}{\partial x_{j}}\left(\rho u_{i} u_{j}\right)-g_{i}\right)^{n} \mathrm{~d} \Omega-\int_{\Omega} \frac{\partial \tilde{W}_{i}}{\partial x_{j}} \tau_{i j}^{n} \mathrm{~d} \Omega \\
& +\int_{\Gamma} \tilde{W}_{i} n_{j} \tau_{i j}^{n} \mathrm{~d} \Gamma+\frac{\Delta t}{2} \int_{\Omega} \frac{\partial}{\partial x_{k}}\left(u_{k}^{n} \tilde{W}_{i}\right) R_{i}^{n} \mathrm{~d} \Omega
\end{aligned}
$$


where $\mathbf{n}$ is the unit outward normal to $\Gamma$ and we have assumed that $R_{i}^{n}=0$ on $\Gamma$ for the last term in (10) after integration by parts.

Boundary conditions expressed in terms of traction can be (weakly) prescribed in (13). Apart from the prescription of the momentum itself (directly or by imposition of the velocity), we consider the following possibilities of boundary conditions:

(a) the whole traction prescribed on $\Gamma_{\mathrm{T}}:-p n_{i}+n_{j} \tau_{i j}=t_{i}$ (given)

(b) only the pressure component of the traction prescribed on $\Gamma_{\mathrm{P}}:-p n_{i}=t_{i}^{\mathrm{p}}$ (given)

(c) the free part of the boundary, $\Gamma_{\mathrm{F}}$.

Conditions (a) and (b) are standard, especially (a). However, condition (c) is not as clear as the others. The idea is to leave $\Gamma_{\mathrm{F}}$ free, without any prescription either on the velocity or on the traction or part of it. This approach has been commonly used in compressible flow problems at supersonic outflows, but can be used as an outflow boundary condition for other types of flow. ${ }^{14}$

The prescription of boundary conditions (a) in (13) yields

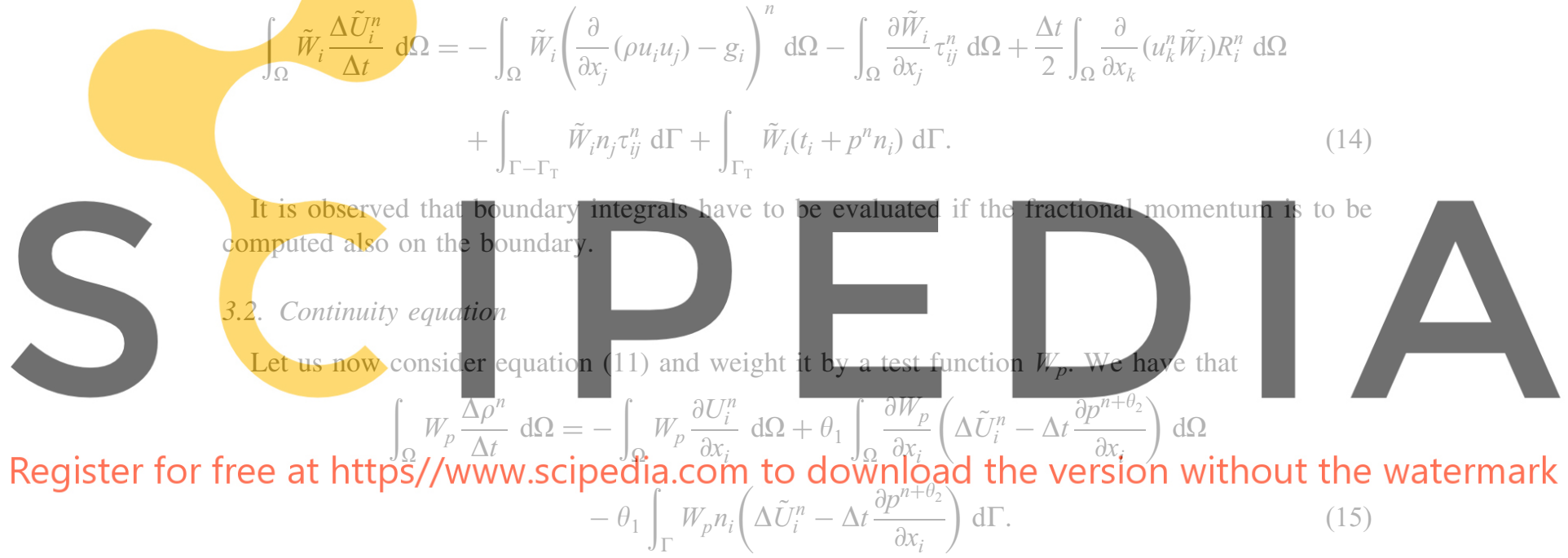

As a boundary condition, we impose that the normal component of (12) be also verified on $\Gamma$, a condition equivalent to imposing that the normal component of the momentum (equation (7)) be verified on $\Gamma$. This leads to

$$
n_{i}\left(\Delta \tilde{U}_{i}^{n}-\Delta t \frac{\partial p^{n+\theta_{2}}}{\partial x_{i}}\right)=n_{i} \Delta U_{i}^{n}
$$

on the part of the boundary $\Gamma_{\mathrm{C}}$ where the test function for the continuity equation, $W_{p}$, does not vanish. Observe that for the semi-implicit case that we consider here $\left(\theta_{2}>0\right)$ both the pressure and the density appear in (15). Either of these can be chosen as the variable for the continuity equation, as will be discussed in the following section. Thus $\Gamma_{\mathrm{C}}$ is the part of $\Gamma$ where either $p$ or $\rho$ is free, depending on which variable is used. Suppose for example that the choice is $p$. According to the type of boundary conditions above, we have that

(a) on $\Gamma_{\mathrm{T}}: p=n_{i} \tau_{i j} n_{j}-n_{i} t_{i}$

(b) on $\Gamma_{\mathrm{P}}: p=n_{i} t_{i}^{\mathrm{p}}$.

In both cases we have a Dirichlet type of boundary condition for the pressure, so that $W_{p}=0$ on that part of $\Gamma$ and $\Gamma_{\mathrm{C}}=\Gamma-\Gamma_{\mathrm{T}}-\Gamma_{\mathrm{P}}$. On the other hand, $\Delta U_{i}^{n}$ is also known on the part of the 
boundary where the momenum is given. The problem arises on $\Gamma_{\mathrm{F}}$, i.e. for condition (c) stated above. In this case neither $W_{p}=0$ nor $\Delta U_{i}^{n}$ is known. If equation (16) is used in the boundary integral of (15), we obtain an equation that involves $U_{i}^{n+1}$, which is not yet known. Therefore this equation becomes coupled with the weak form of (12) discussed next. In order to avoid this coupling, we take $n_{i} \Delta U_{i}^{n}$ as zero. For transient calculations, if the normal component of the momentum varies on $\Gamma_{\mathrm{F}}$, this will be an approximation of order $\Delta t$. In any case the steady state solution (if reached) will be correct. Recall that this approximation is needed only when $\Gamma_{\mathrm{F}}$ is not empty, i.e. when the nonstandard boundary condition (c) is used.

Let $\Gamma_{\mathrm{D}}$ be the part of $\Gamma$ where the momentum is known. Using equation (16) and the approximation just described, equation (15) can be written as

$$
\int_{\Omega} W_{p} \frac{\Delta \rho^{n}}{\Delta t} \mathrm{~d} \Omega=-\int_{\Omega} W_{p} \frac{\partial U_{i}^{n}}{\partial x_{i}} \mathrm{~d} \Omega+\theta_{1} \int_{\Omega} \frac{\partial W_{p}}{\partial x_{i}}\left(\Delta \tilde{U}_{i}^{n}-\Delta t \frac{\partial p^{n+\theta_{2}}}{\partial x_{i}}\right) \mathrm{d} \Omega-\theta_{1} \int_{\Gamma_{\mathrm{D}}} W_{p} n_{i} \Delta U_{i}^{n} \mathrm{~d} \Gamma .
$$

This is the weak form of the continuity equation that we use if the unknown is either the pressure or the density. In the second case the pressure may be considered known where the density is given by using the equation of state and a guess for the temperature, if required.

\subsection{Momentum equation}

Finally, for (12) we have that
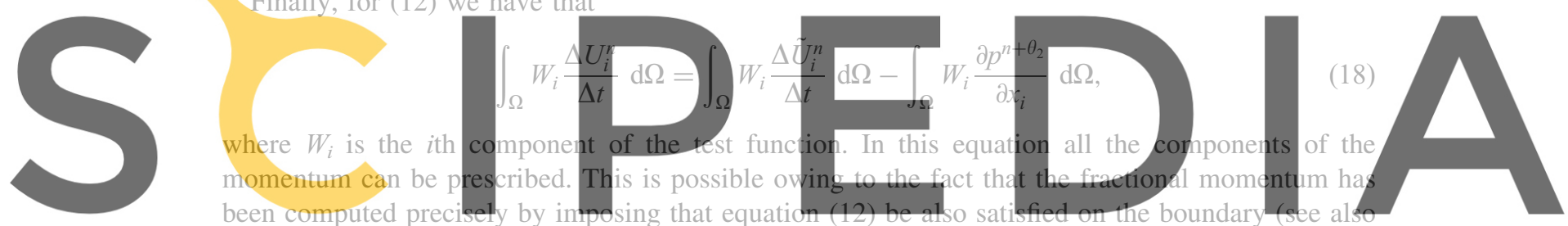

the comment about this in Section 2)

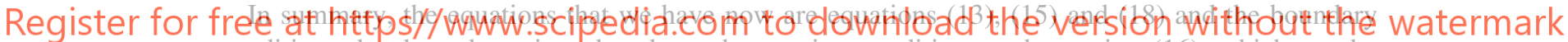

conditions that have been introduced are the traction conditions and equation (16), which can be considered as the normal component of the momentum equations. Moreover, since the fractional momentum is also computed on the boundary, all the components of the momentum itself can be prescribed on it. However, the momentum is usually not directly fixed for compressible flows, but instead the velocity is given as boundary condition. We use the common approach of taking the momentum as prescribed using the given velocity values and the density computed in the current time step. This prescription is performed at the end of this step.

\subsection{Energy equation}

Once equations (13), (15) and (18) are solved, we have the momentum and either the pressure or the density at the current time step. It remains to compute the total energy. For that we can solve explicitly or implicitly the last scalar equation in the vector equation (1). Using the former option with a discretization along the characteristics, we have that

$$
\frac{\Delta E^{n}}{\Delta t}=R_{\mathrm{E}}^{n}-\frac{\Delta t}{2} u_{k}^{n} \frac{\partial R_{\mathrm{E}}^{n}}{\partial x_{k}},
$$

where $R_{\mathrm{E}}$ is defined as

$$
R_{\mathrm{E}}:=-\frac{\partial}{\partial x_{i}}\left(u_{i}(E+p)-k \frac{\partial T}{\partial x_{i}}-\tau_{i j} u_{j}\right)
$$


Weighting this equation by a test function $W_{\mathrm{E}}$, integrating the diffusion and heat production terms by parts, setting $R_{\mathrm{E}}=0$ on the boundary and prescribing the total heat flux (from production and conduction) as $H$ on a part of the boundary $\Gamma_{\mathrm{H}}$, we get

$$
\begin{aligned}
\int_{\Omega} W_{\mathrm{E}} \frac{\Delta E^{n}}{\Delta t} \mathrm{~d} \Omega= & -\int_{\Omega} W_{\mathrm{E}} \frac{\partial}{\partial x_{i}}\left[u_{i}(E+p)\right]^{n} \mathrm{~d} \Omega-\int_{\Omega} \frac{\partial W_{\mathrm{E}}}{\partial x_{i}}\left(k \frac{\partial T}{\partial x_{i}}+\tau_{i j} u_{j}\right)^{n} \mathrm{~d} \Omega \\
& +\frac{\Delta t}{2} \int_{\Omega} \frac{\partial}{\partial x_{k}}\left(u_{k}^{n} W_{\mathrm{E}}\right) R_{\mathrm{E}}^{n} \mathrm{~d} \Omega+\int_{\Gamma_{\mathrm{H}}} W_{\mathrm{E}} H \mathrm{~d} \Gamma .
\end{aligned}
$$

On $\Gamma-\Gamma_{\mathrm{H}}$ we assume that $W_{\mathrm{E}}=0$, i.e. the energy is known there. As for the momentum, the total energy is not normally prescribed, but instead of this the temperature is given. In this case we prescribe the total energy using the already known values of velocity and density and the prescribed temperatures.

If the solution of the flow equations has no shocks, instead of the energy equation written in conservation form one can solve the heat equation

$$
\frac{\partial T}{\partial t}=R_{\mathrm{T}}:=-u_{i} \frac{\partial T}{\partial x_{i}}+\frac{1}{C_{\mathrm{v}} \rho} \frac{\partial}{\partial x_{i}}\left(k \frac{\partial T}{\partial x_{i}}\right)+\frac{1}{C_{\mathrm{v}} \rho} \sigma_{i j} \frac{\partial u_{i}}{\partial x_{j}} .
$$

Usually, this equation is written with the heat capacity $C_{\mathrm{v}} \rho$ multiplying the temporal derivative of the

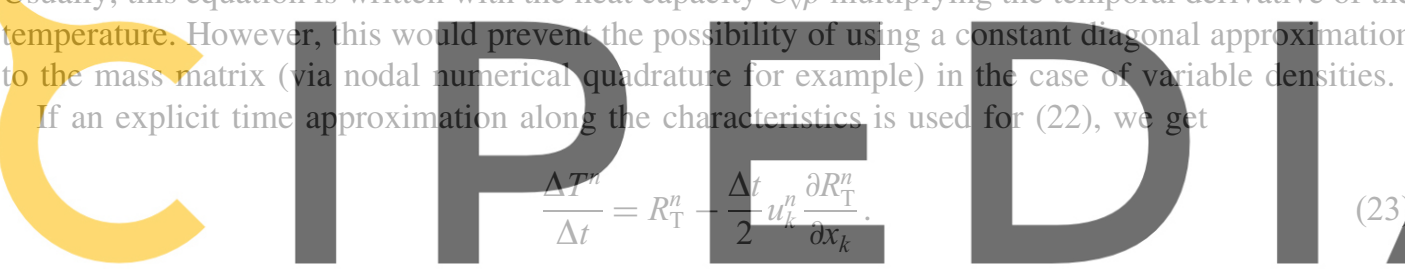

Let us now weight this equation by a test function $W_{\mathrm{T}}$, integrate the diffusion term by parts, set $R_{\mathrm{T}}=0$

$$
\begin{aligned}
\int_{\Omega} W_{\mathrm{T}} \frac{\Delta T^{n}}{\Delta t} \mathrm{~d} \Omega= & \int_{\Omega} W_{\mathrm{T}}\left[\left(-u_{i}+\frac{k}{C_{\mathrm{v}} \rho^{2}} \frac{\partial \rho}{\partial x_{i}}\right) \frac{\partial T}{\partial x_{i}}+\frac{1}{C_{\mathrm{v}} \rho} \sigma_{i j} \frac{\partial u_{i}}{\partial x_{j}}\right]^{n} \mathrm{~d} \Omega-\int_{\Omega} \frac{\partial W_{\mathrm{T}}}{\partial x_{i}}\left(\frac{k}{C_{\mathrm{v}} \rho} \frac{\partial T}{\partial x_{i}}\right)^{n} \mathrm{~d} \Omega \\
& +\frac{\Delta t}{2} \int_{\Omega} \frac{\partial}{\partial x_{k}}\left(u_{k}^{n} W_{\mathrm{T}}\right) R_{\mathrm{T}}^{n} \mathrm{~d} \Omega+\int_{\Gamma_{\mathrm{H}}} \frac{1}{C_{\mathrm{v}} \rho^{n}} W_{\mathrm{T}} H \mathrm{~d} \Gamma .
\end{aligned}
$$

The temperature is assumed to be known on $\Gamma-\Gamma_{\mathrm{H}}$.

\section{DISCRETE PROBLEM AND SOLUTION STRATEGIES}

With the weak form of the differential equations already established, we can proceed to discretize the space. We do this using the standard Galerkin method, since the term coming from the discretization in time along the characteristics will stabilize the convective terms. This means that we take all the test functions $\tilde{W}_{i}, W_{p}, W_{i}, W_{\mathrm{E}}$ and $W_{\mathrm{T}}$ equal to the shape functions. Also, some additional shockcapturing viscosity will be needed in the presence of discontinuities or sharp gradients of the solution. The method we use is based on the ideas presented in Reference 15 and explained in Part I of this paper $^{3}$ and thus we shall not describe it here. 
Let us first consider the equations for the fractional momentum (14) and the end-of-step momentum (18). Once the spatial discretization has been performed, the discrete version of these equations can be written in matrix form, the structure of which is

$$
\begin{aligned}
\mathbf{M} \frac{\Delta \tilde{\overline{\mathbf{U}}}^{n}}{\Delta t} & =\mathbf{F}_{1}-\mathbf{K} \overline{\mathbf{U}}^{n}, \\
\mathbf{M}_{0} \frac{\Delta \overline{\mathbf{U}}_{0}^{n}}{\Delta t} & =\mathbf{M}_{0} \frac{\Delta \tilde{\overline{\mathbf{U}}}_{0}^{n}}{\Delta t}-\mathbf{G}_{0} \overline{\mathbf{p}}^{n+\theta_{2}}+\mathbf{F}_{2} .
\end{aligned}
$$

Vectors of nodal unknowns have been indicated by a boldface character and an overbar. Matrices $\mathbf{M}$, $\mathbf{K}$ and $\mathbf{G}$ are the standard mass matrix for vector fields, the matrix coming from the viscous and convective terms in the equation for the fractional momentum and the matrix coming from the gradient operator respectively. Subscript zero in the previous equations refers to not prescribed degrees of freedom for the momentum (in the sense indicated above) and $\mathbf{F}_{2}$ contains precisely the contribution from $\Delta \overline{\mathbf{U}}^{n}$ and $\Delta \overline{\mathbf{U}}^{n}$ corresponding to the prescribed degrees of freedom for the latter. Here and below we use $\mathbf{F}$ with subscripts to denote a vector which is known at the moment of solving a particular equation.

The discrete version of the energy equation written in conservation form (21) or the heat equation (24) can be solved at the beginning or the end of the time step. These equations have the structure
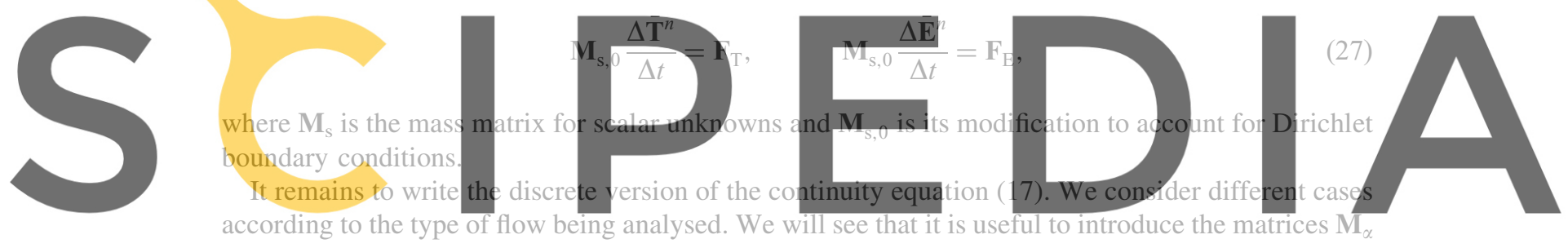

according to the type of flow being analysed. We will see that it is useful to introduce the matrices $\mathbf{M}_{2}$ and $\mathbf{L}_{\beta}$ with components

\section{Register for free at https//www.scipedia.com to download the

$$
M_{\alpha, i j}=\int_{\Omega} \alpha N_{i} N_{j} \mathrm{~d} \Omega, \quad L_{\beta, i j}=\int_{\Omega} \beta \frac{\partial N_{i}}{\partial x_{k}} \frac{\partial N_{j}}{\partial x_{k}} \mathrm{~d} \Omega,
$$

where $N_{i}$ is the shape function associated with the $i$ th node of the finite element mesh with which we assume that all the variables are interpolated and $\alpha$ and $\beta$ are functions that depend on the type of flow.

\subsection{Incompressible and slightly compressible flows}

These two types of flows can be defined by the relation

$$
\Delta \rho^{n}=\alpha \Delta p^{n},
$$

with $\alpha=0$ for fully incompressible flows and $\alpha=1 / c^{2}$ (a positive constant) for slightly compressible flows. In this case, equation (17) can be written as

$$
\begin{aligned}
\int_{\Omega} \alpha W_{p} \frac{\Delta p^{n}}{\Delta t} \mathrm{~d} \Omega+\theta_{1} \Delta t \int_{\Omega} \frac{\partial W_{p}}{\partial x_{i}} \frac{\partial p^{n+\theta_{2}}}{\partial x_{i}} \mathrm{~d} \Omega= & -\int_{\Omega} W_{p} \frac{\partial U_{i}^{n}}{\partial x_{i}} \mathrm{~d} \Omega+\theta_{1} \int_{\Omega} \frac{\partial W_{p}}{\partial x_{i}} \Delta \tilde{U}_{i}^{n} \mathrm{~d} \Omega \\
& -\theta_{1} \int_{\Gamma_{\mathrm{D}}} W_{p} n_{i} \Delta U_{i}^{n} \mathrm{~d} \Gamma .
\end{aligned}
$$


Once the finite element discretization of this equation has been done, the matrix form of the discrete problem is

$$
\mathbf{M}_{\alpha} \frac{\Delta \overline{\mathbf{p}}^{n}}{\Delta t}+\theta_{1} \Delta t \mathbf{L}_{\beta} \overline{\mathbf{p}}^{n+\theta_{2}}=\mathbf{F}_{\mathrm{C}}
$$

with $\alpha$ the parameter appearing in (29) and $\beta=1$ in this case. In (31) we have introduced

$$
\mathbf{F}_{\mathrm{C}}:=-\mathbf{D} \overline{\mathbf{U}}^{n}+\theta_{1} \mathbf{G}^{\mathrm{T}} \Delta \tilde{\overline{\mathbf{U}}}^{n}+\mathbf{F}_{\mathrm{D}},
$$

where $\mathbf{F}_{\mathrm{D}}$ is the vector coming from the last term in (30), i.e. from the boundary values of the momentum, and $\mathbf{D}$ is the matrix coming from the divergence operator. Dirichlet boundary conditions for the pressure are assumed to be included in (31).

Of snecial interest is the case of fully incompressible flows, i.e. $\alpha=0$. It is well known that in this case the velocity and pressure finite element interpolations must satisfy the Babuška-Brezzi conditions when the classical $\mathbf{u}-p$ approach is used. This is not the case using the type of fractional step methods that we are considering. We justify this in the following. To simplify the discussion, we assume that $\mathbf{U}$ is prescribed as zero on the whole boundary $\Gamma$.

Omitting the subscript $\beta$ for a moment (it is one), the matrix form of (25), (26) and (31) can be written as
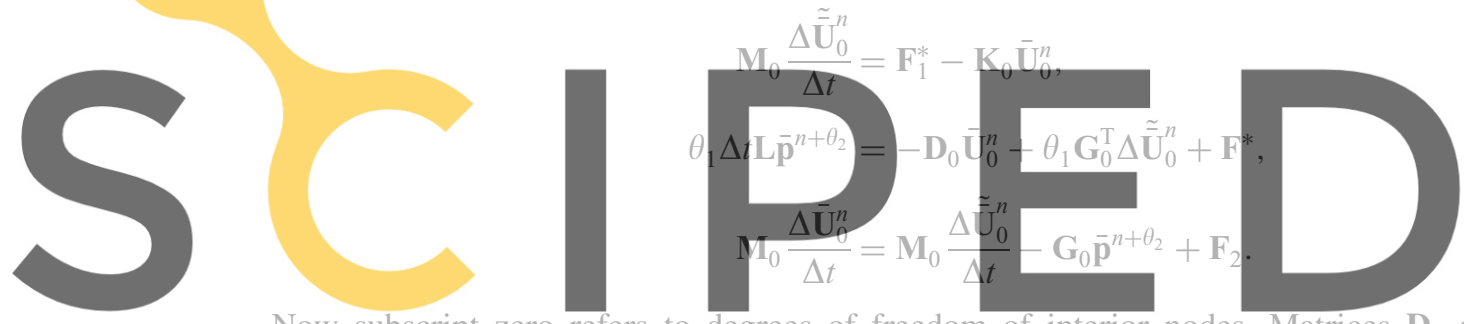

Now subscript zero refers to degrees of freedom of interior nodes. Matrices $\mathbf{D}_{0}$ and $\mathbf{G}_{0}^{\mathrm{T}}$ are the submatrices of $\mathbb{D}$ and $\mathbf{G}^{\mathrm{T}}$ corresponding to these nodes. They are related by $\mathbb{D}_{0}=-\mathbf{G}_{0}^{\mathrm{T}}$. Vectors $\mathbb{F}_{1}^{*}$ From (35) we get that

$$
\Delta \tilde{\mathbf{U}}_{0}^{n}=\Delta \overline{\mathbf{U}}_{0}^{n}+\Delta t \mathbf{M}_{0}^{-1} \mathbf{G}_{0} \overline{\mathbf{p}}^{n+\theta_{2}}-\Delta t \mathbf{M}_{0}^{-1} \mathbf{F}_{2} .
$$

Using this in (33) and (34), we obtain

$$
\begin{gathered}
\mathbf{M}_{0} \frac{\Delta \overline{\mathbf{U}}_{0}^{n}}{\Delta t}+\mathbf{K}_{0} \overline{\mathbf{U}}_{0}^{n}+\mathbf{G}_{0} \overline{\mathbf{p}}^{n+\theta_{2}}=\mathbf{F}_{1}^{*}+\mathbf{F}_{2}, \\
\mathbf{D}_{0} \overline{\mathbf{U}}_{0}^{n+\theta_{1}}+\theta_{1} \Delta t\left(\mathbf{L}-\mathbf{G}_{0}^{\mathrm{T}} \mathbf{M}_{0}^{-1} \mathbf{G}_{0}\right) \overline{\mathbf{p}}^{n+\theta_{2}}=\mathbf{F}_{\mathrm{C}}^{*},
\end{gathered}
$$

with

$$
\mathbf{F}_{\mathrm{C}}^{*}:=\mathbf{F}^{*}-\theta_{1} \Delta t \mathbf{G}_{0}^{\mathrm{T}} \mathbf{M}_{0}^{-1} \mathbf{F}_{2} .
$$

Clearly we must have $\theta_{1}>0$ and $\theta_{2}>0$ in order to have a solvable problem.

The important point in (38) is the presence of the matrix $\mathbf{B}:=\mathbf{L}-\mathbf{G}_{0}^{\mathrm{T}} \mathbf{M}_{0}^{-1} \mathbf{G}_{0}$, which can be understood as the difference between two discrete Laplacian operators. This matrix provides additional stability and in particular allows us to use equal velocity-pressure finite element interpolations in the incompressible case, as had been noticed for example in References 16 and 17. This is so because this matrix is positive semidefinite. Let us prove this and for that let us denote by $V_{h}$ the finite element space to interpolate $\mathbf{U}$ with homogeneous boundary conditions on $\Gamma$ and by 
$Q_{h} \subset C^{0}(\Omega)$ the finite element space for $p$. We can consider the vector space $E_{h}:=V_{h}+\nabla Q_{h}$, where $\nabla Q_{h}$ denotes the space of vector functions which are gradients of functions in $Q_{h}$. It can be split as

$$
E_{h}=V_{h} \oplus V_{h}^{\perp}=\operatorname{span}\left\{\mathbf{v}_{1}, \ldots, \mathbf{v}_{n}\right\} \oplus \operatorname{span}\left\{\mathbf{v}_{1}^{\prime}, \ldots, \mathbf{v}_{m}^{\prime}\right\}
$$

We must prove that

$$
\overline{\mathbf{p}}^{\mathrm{T}} \mathbf{B} \overline{\mathbf{p}}=\overline{\mathbf{p}}^{\mathrm{T}} \mathbf{L} \overline{\mathbf{p}}-\overline{\mathbf{p}}^{\mathrm{T}} \mathbf{G}_{0}^{\mathrm{T}} \mathbf{M}_{0}^{-1} \mathbf{G}_{0} \overline{\mathbf{p}}
$$

is non-negative. If we consider the decomposition

$$
\nabla p=\mathbf{g}_{1}+\mathbf{g}_{2}=\sum_{k=1}^{n} \bar{g}_{1, k} \mathbf{v}_{k}+\sum_{k=1}^{m} \bar{g}_{2, k} \mathbf{v}_{k}^{\prime}, \quad \mathbf{g}_{1} \in V_{h}, \quad \mathbf{g}_{2} \in V_{h}^{\perp},
$$

we have that

$$
\overline{\mathbf{p}}^{\mathrm{T}} \mathbf{L} \overline{\mathbf{p}}=\int_{\Omega}|\nabla p|^{2} \mathrm{~d} \Omega=\overline{\mathbf{g}}_{1}^{\mathrm{T}} \mathbf{M}_{0} \overline{\mathbf{g}}_{1}+\int_{\Omega} \mathbf{g}_{2} \cdot \mathbf{g}_{2} \mathrm{~d} \Omega
$$

and, on the other hand, if $M_{i j}^{-1}$ are the components of $\mathbf{M}_{0}^{-1}$,
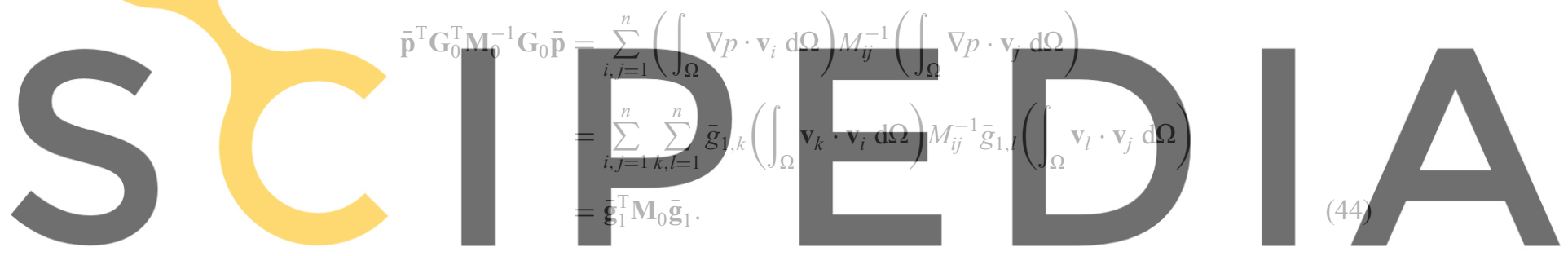

From equations (43) and (44) we obtain

\section{Register for free at https//www.scipedia.com to download the version without the watermark $\overline{\mathbf{p}}^{1} \mathbf{B} \overline{\mathbf{p}}=\int_{\Omega} \mathrm{g}_{2} \cdot \mathrm{g}_{2} \mathrm{~d} \Omega \geqslant 0$.

In general there are only a few components of $\nabla p$ in $V_{h}$ and the matrix $\mathbf{B}$ stabilizes all the pressure components in $V_{h}^{\perp}$. Let us consider for example the linear P1 element and let $n_{\text {sd }}$ be the number of space dimensions. If $\nabla p$ is continuous, it must be globally constant, i.e. $\nabla p$ can only have $n_{\text {sd }}$ components in $V_{h}$.

\subsection{Barotropic flows}

Let us now consider the flow of compressible barotropic fluids; that is to say, fluids for which there is an equation of state that involves only the density and the pressure but not the temperature. In general we write this equation as $p=p(\rho)$, but we will particularize it to the case

$$
p=A \rho^{\gamma}
$$

where $A$ and $\gamma$, the adiabatic exponent, are physical constants. This situation is found for example in the case of isentropic flow of perfect gases.

In the case of incompressible or slightly compressible flows we have formulated the continuity equation in terms of the pressure only. However, now we have the possibility of choosing either the density or the pressure as the unknown of the problem. Let us start with the former option. 


\section{Density as variable}

If we choose to write the continuity equation (17) using the density, we have to express the pressure gradient in terms of the density. For this we use the approximation

$$
\frac{\partial p^{n+\theta_{2}}}{\partial x_{i}}=\left(\frac{\mathrm{d} p}{\mathrm{~d} \rho}\right)^{n} \frac{\partial \rho^{n+\theta_{2}}}{\partial x_{i}}=\frac{\gamma p^{n}}{\rho^{n}} \frac{\partial \rho^{n+\theta_{2}}}{\partial x_{i}} .
$$

The approximation relies on the fact that we evaluate the derivative of $p$ with respect to $\rho$ (the square of the speed of sound) at $n$ instead of at $n+\theta_{2}$. This may be thought of as a linearization of the problem.

Using equation (47) in (17), it is found that the discrete continuity equation can be written in this case as

$$
\mathbf{M}_{\alpha} \frac{\Delta \overline{\boldsymbol{\rho}}^{n}}{\Delta t}+\theta_{1} \Delta t \mathbf{L}_{\beta} \overline{\boldsymbol{\rho}}^{n+\theta_{2}}=\mathbf{F}_{\mathrm{C}}
$$

now with $\alpha=1$ and $\beta=\gamma p^{n} / \rho^{n}$. Observe that this equation has the same structure as (31) but with the density being the unknown instead of the pressure.

\section{Pressure as variable}

If instead of using the density we use the pressure, the approximation that we employ is

$$
\Delta \rho^{n}=\left(\frac{\mathrm{d} \rho}{\mathrm{d} p}\right)^{n} \Delta p^{n}
$$

which is of order $O\left(\left(\Delta p^{n}\right)^{2}\right)$. This approximation leads to

$$
\frac{\Delta \rho^{n}}{\Delta t}=\left(\frac{\mathrm{d} \rho}{\mathrm{d} p}\right)^{n} \frac{\Delta p^{n}}{\Delta t}=\frac{\rho^{n}}{\gamma p^{n}} \frac{\Delta p^{n}}{\Delta t}
$$

and the discrete continuity equation can now be written again as

$$
\mathbf{M}_{\alpha} \frac{\Delta \overline{\mathbf{p}}^{n}}{\Delta t}+\theta_{1} \Delta t \mathbf{L}_{\beta} \overline{\mathbf{p}}^{n+\theta_{2}}=\mathbf{F}_{\mathrm{C}}
$$

i.e. exactly as (31) but now with $\alpha=\rho^{n} /\left(\gamma p^{n}\right)$ and $\beta=1$.

Having written the discrete continuity equation, the algorithm within each time step for the case of barotropic flows is as follows.

1. Solve for the fractional momentum (equation (25)).

2. Solve the continuity equation (51) for $p^{n+1}$ (or (48) for $\rho^{n+1}$ ).

3. Obtain $\rho^{n+1}$ from the equation of state (46) (or $p^{n+1}$ if $\rho^{n+1}$ has been used in step 2).

4. Solve for the end-of-step momentum (equation (26)).

As mentioned before, the energy (or the heat) equation may be solved at the beginning or the end of the time step. In this particular case this equation is uncoupled from the others.

\subsection{Perfect gases}

In this case the equation of state involves not only the pressure and the density but also the temperature. This equation is

$$
p=\rho R T
$$


where $R$ is the universal gas constant. The appearance of the temperature in this equation complicates a little the treatment of the continuity equation. As before, we may use either the density or the pressure as variable.

\section{Density as variable}

In this case we need to relate the pressure gradient to the density if $\theta_{2}>0$ in (17), the situation that we consider throughout. We have that

$$
\frac{\partial p^{n+\theta_{2}}}{\partial x_{i}}=\frac{\partial \rho^{n+\theta_{2}}}{\partial x_{i}} R T^{n+\theta_{2}}+\rho^{n+\theta_{2}} R \frac{\partial T^{n+\theta_{2}}}{\partial x_{i}} .
$$

If we use this expression directly in (17), the continuity equation will be coupled to the energy (or heat) conservation equation. In order to avoid this, we use an iterative strategy based on assuming that $T^{n+\theta_{2}}$ is known and then correcting it. There is also another aspect that is computationally inconvenient. If we take $\rho^{n+\theta_{2}}$ as unknown in the second term of the RHS of (53), this will lead to a non-symmetric matrix (see equation (17)). This can be circumvented if we also assume that $\rho^{n+\theta_{2}}$ is known and then we correct it.

Let $T_{\mathrm{g}}$ be a guess for $T^{n+\theta_{2}}$ within the time step under consideration and $\rho_{\mathrm{g}}$ a guess for $\rho^{n+\theta_{2}}$. Equation (33) may be replaced by

$$
\frac{\partial p^{n+\theta_{2}}}{\partial x_{i}}=\frac{\partial \rho^{n+\theta_{2}}}{\partial x_{i}} R T_{\mathrm{g}}+\rho_{\mathrm{g}} R \frac{\partial T_{\mathrm{g}}}{\partial x_{i}}
$$

The second term in this equation contributes to the RHS of the discrete continuity equation. If we denote this contribution by $\mathbf{F}_{\rho}$, this discrete equation is

$$
\mathbf{M}_{\alpha} \frac{\Delta \overline{\boldsymbol{\rho}}^{n}}{\Delta t}+\theta_{1} \Delta t \mathbf{L}_{\beta} \overline{\boldsymbol{\rho}}^{n+\theta_{2}}=\mathbf{F}_{\mathrm{C}}+\mathbf{F}_{\rho}
$$

with $\alpha=1$ and $\beta=R T_{\mathrm{g}}$. This equation is similar to (48). Apart from the coefficients $\alpha$ and $\beta$, the only difference is the term $\mathbf{F}_{\rho}$, which comes from the spatial derivative of the temperature.

\section{Pressure as variable}

As for the case of barotropic flows, we may also use the pressure as the unknown of the continuity equation. For that we only need to use the equation of state (52), from which we have

$$
\rho^{n+1}=\frac{p^{n+1}}{R T^{n+1}} .
$$

As in the previous case, we need to guess the value of $T^{n+1}$ by $T_{\mathrm{g}}$ in order to uncouple the resulting continuity equation and the energy equation. We may then write

$$
\Delta \rho^{n}=\frac{\Delta p^{n}}{R T_{\mathrm{g}}}+\left(\frac{p^{n}}{R T_{\mathrm{g}}}-\frac{p^{n}}{R T^{n}}\right)
$$

The term in parentheses contributes to the RHS of the discrete continuity equation with a vector $\mathbf{F}_{p}$. This equation can be written as

$$
\mathbf{M}_{\alpha} \frac{\Delta \overline{\mathbf{p}}^{n}}{\Delta t}+\theta_{1} \Delta t \mathbf{L}_{\beta} \overline{\mathbf{p}}^{n+\theta_{2}}=\mathbf{F}_{\mathrm{C}}+\mathbf{F}_{p}
$$

with $\alpha=1 / R T_{\mathrm{g}}$ and $\beta=1$. Again this equation has the same structure as (51) but with a modification of the RHS due to the variation (now in time) of the temperature. 
From numerical experiments we have found that this approach does not work well in the presence of strong shocks, in the sense that we have not been able to obtain a converged steady state solution in these cases. We attribute this to the appearance of the temperature as a denominator in the function $\alpha$. This makes the coefficients of $\mathbf{M}_{\alpha}$ difficult to evaluate numerically and with possibly high variations from one time step to the next in the vicinity of shocks.

If we use either the pressure or the density as unknown, within each time step we need to use an iterative scheme to correct the temperature that has been guessed. This iterative scheme is as follows.

1. Solve the energy equation or the heat equation (equation (27)).

2. Solve for the fractional momentum (equation (25)).

3. Guess a temperature $T_{\mathrm{g}}$.

4. Solve the continuity equation (58) for $p^{n+1}$ (or (55) for $\rho^{n+1}$ ).

5. Obtain $\rho^{n+1}$ from the equation of state (56) (or $p^{n+1}$ if $\rho^{n+1}$ has been used in step 4).

6. Solve for the end-of-step momentum (equation (26)).

7. Correct $T_{\mathrm{g}}$ using $T^{n+1}$ and $\rho_{\mathrm{g}}$ using $\rho^{n+1}$, if needed.

8. Check convergence. If not satisfactory, go to step 4.

Let us make some remarks about this algorithm.

(a) Use the heat equation in step 1. If this is done, we already have $T^{n+1}$ and therefore there is no need to iterate at all. However, we have found that this approach may yield wrong results in the presence of shocks, with a wrong location for them and/or without satisfying the jump conditions. It is well known that in this situation it is necessary to use the energy equation written in conservation form. By doing this, after step 1 we have $E^{n+1}$. A natural way to compute $T_{\mathrm{g}}$ is to use this and the density and velocity of the previous time step.

(b) Do not check convergence; that is to say, take $T_{\mathrm{g}}$ computed as indicated before as $T^{n+1}$ in the continuity equation and also $\rho_{\mathrm{g}}$ as $\rho^{n+1}$ in the vector $\mathbf{F}_{\rho}$ if the density is used as unknown. This is an approximation of order $O(\Delta t)$ that works well if only the steady state is of interest.

(c) The steady state is reached slightly faster and time steps slightly larger can be used if a couple of iterations of the previous scheme are performed. We have found almost no difference either in the numerical results or in the convergence behaviour if more than two iterations are done.

Before closing this section, let us remark that for all the types of flows considered we have written the continuity equation in a very similar way. Using the pressure as variable, the general form is

$$
\mathbf{M}_{\alpha} \frac{\Delta \overline{\mathbf{p}}^{n}}{\Delta t}+\theta_{1} \Delta t \mathbf{L}_{\beta} \overline{\mathbf{p}}^{n+\theta_{2}}=\mathbf{F}_{\mathrm{C}}^{\prime}
$$

with $\beta=1$,

$$
\alpha= \begin{cases}0 & \text { for incompressible flows } \\ 1 / c^{2} & \text { for slightly compressible flows } \\ \rho^{n} / \gamma p^{n} & \text { for barotropic flows (isentropic perfect gases) } \\ 1 / R T_{\mathrm{g}} & \text { for perfect gases }\end{cases}
$$

and $\mathbf{F}_{\mathrm{C}}^{\prime}=\mathbf{F}_{\mathrm{C}}$, except in the case of perfect gases for which $\mathbf{F}_{\mathrm{C}}^{\prime}=\mathbf{F}_{\mathrm{C}}+\mathbf{F}_{p}$.

The density can be used as variable only for barotropic fluids and perfect gases. In this case the discrete continuity equation is

$$
\mathbf{M}_{\alpha} \frac{\Delta \overline{\boldsymbol{\rho}}^{n}}{\Delta t}+\theta_{1} \Delta t \mathbf{L}_{\beta} \overline{\boldsymbol{\rho}}^{n+\theta_{2}}=\mathbf{F}_{\mathrm{C}}^{\prime}
$$


now with $\alpha=1$,

$$
\beta= \begin{cases}\gamma p^{n} / \rho^{n} & \text { for barotropic flows (isentropic perfect gases) } \\ R T_{\mathrm{g}} & \text { for perfect gases }\end{cases}
$$

and $\mathbf{F}_{\mathrm{C}}^{\prime}=\mathbf{F}_{\mathrm{C}}$ for barotropic fluids and $\mathbf{F}_{\mathrm{C}}^{\prime}=\mathbf{F}_{\mathrm{C}}+\mathbf{F}_{\rho}$ for perfect gases.

In all cases the matrix of the algebraic system of equations to be solved is symmetric and positive definite (for incompressible confined flows a pressure needs to be specified). We use the conjugate gradient method to solve it. In general, very few iterations are needed for convergence, since the unknown at the previous time step is a good initial guess for its value at the current one.

\section{NUMERICAL EXAMPLES}

In this section we present the numerical solution that we have obtained for a classical benchmark problem for three very different types of flow, all using the formulation described in the previous section for each case.

\subsection{Incompressible flow in a cavity}

This is a classical test problem to evaluate the behaviour of any algorithm devised to numerically solve incompressible flows. A viscous fluid is confined in a square cavity while one of its edges slides tangentially, where the horizontal component of the velocity is prescribed as 1.0 and the normal component as $0 \cdot 0$. On the rest of the cavity edges the no-slip condition is used. The zero of the pressure is fixed at one node of the bottom edge. The no-leak condition is used at the top right and left corners, allowing the velocity to decrease linearly in the end elements in the tangential direction.

The domain is discretized in a structured mesh made of 2888 P1 elements, slightly refined from the centre to the edges (Figure 1). There are 1521 nodal points. The results are shown in Figures 2 $(R e=1000)$ and $3(R e=5000)$.

These results are compared with those obtained by Ghia et al. ${ }^{19}$ in Figure 4, showing the velocity $x$-component along a vertical central cut.

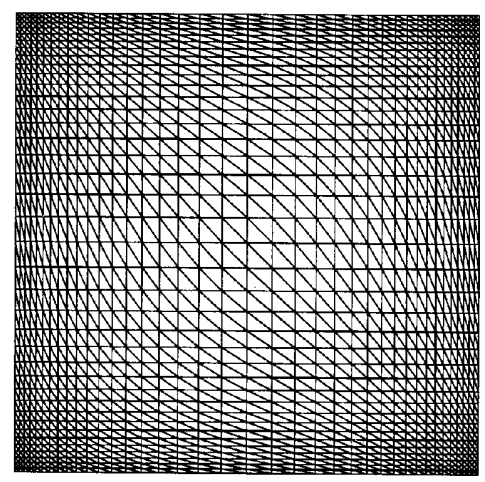

Figure 1. Driven cavity flow: Mesh 

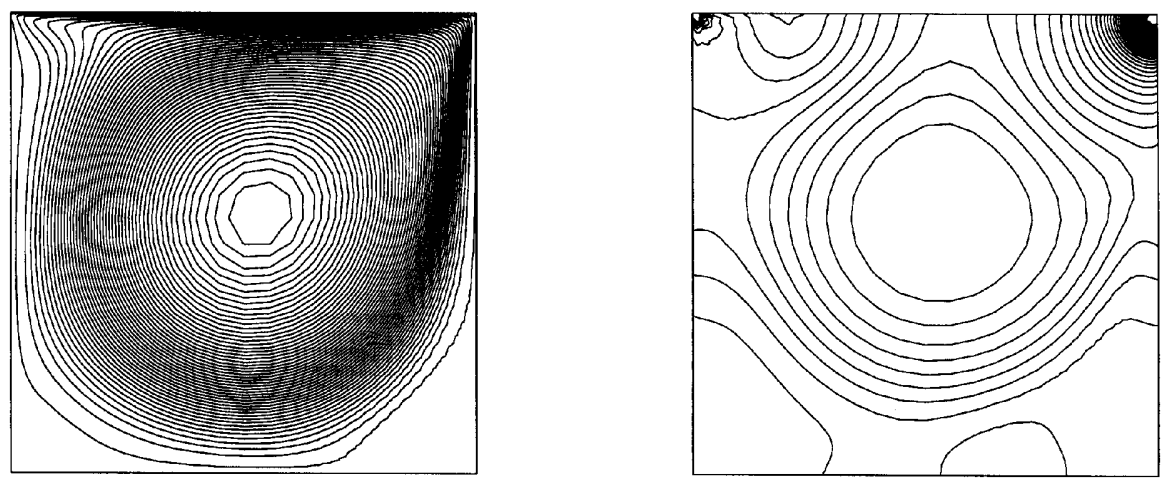

Figure 2. Driven cavity flow: streamlines (left) and pressure contours (right) at $R e=1000$
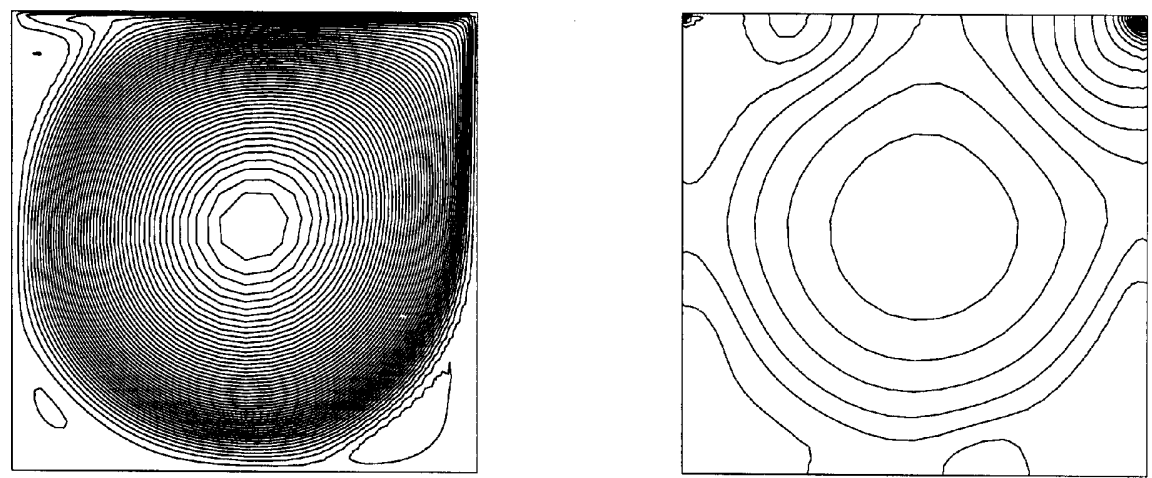

Figure 3. Driven cavity flow: streamlines (left) and pressure contours (right) at $R e=5000$
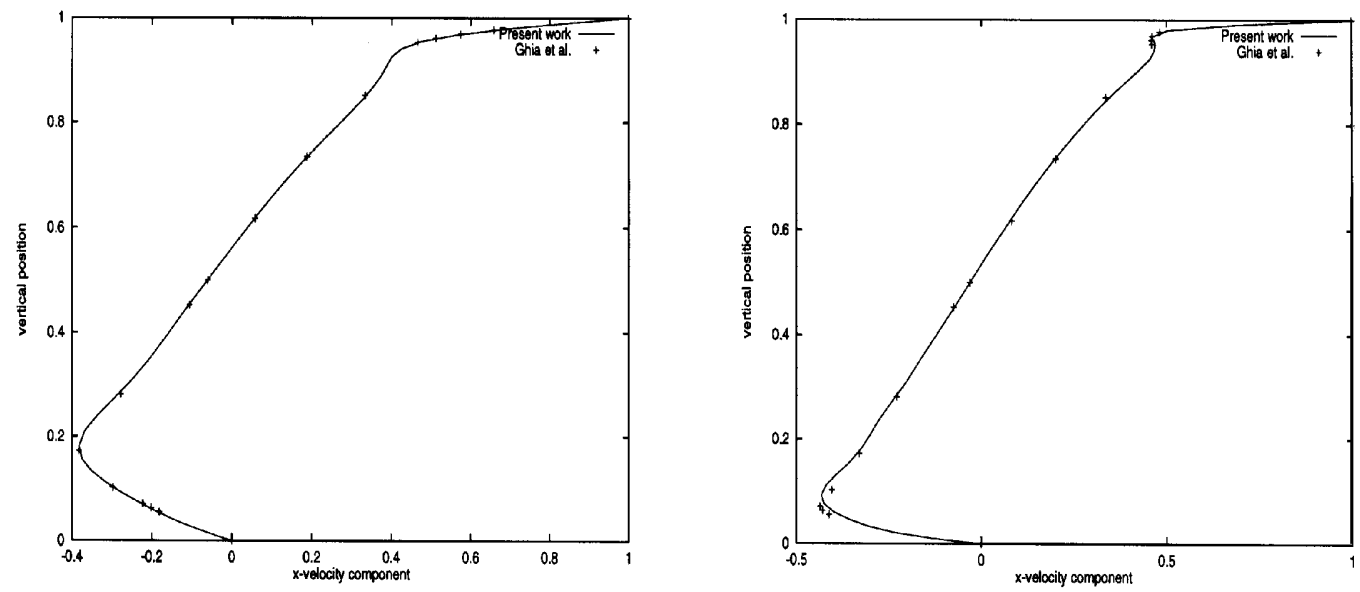

Figure 4. Driven cavity flow: horizontal velocity component at $R e=1000$ (left) and 5000 (right) 


\subsection{Inviscid subsonic flow over a NACA 0012 profile}

This example illustrates the behaviour of the algorithm in the case of inviscid subsonic flow with a barotropic state law. The mesh is a rather coarse unstructured one made of 2556 nodes arranged in 4902 P1 elements. Partial views of it are shown in Figure 5.

The angle of attack is $\alpha=0^{\circ}$. The Mach number at the inflow is $M_{\infty}=0 \cdot 5$. The velocity is prescribed at the inflow as 1.0 in the $x$-direction and 0.0 in the $y$-direction. The density at the outflow, $\rho_{\infty}$, is fixed as 1.0. Also, the normal component of the velocity is fixed as 0.0 on the profile. The adiabatic exponent $\gamma$ is 1.4 and the constant $A$ is 2.857136 . Pressure isolines are shown in Figure 6.

In the example shown here, the continuity equation is solved with the pressure as unknown, which gives a slightly better solution (particularly around the stagnation point) than when the density is chosen. Both schemes, however, give acceptable results. Despite the subsonic character of the problem, an additional shock-capturing diffusion is needed, probably owing to the strong gradients present in the solution. As the flow is subsonic throughout the whole domain, we let this artificial diffusion act only in the fractional momentum equation using an algorithmic constant lower than the optimal one. According to reference 15 , its optimal value is 0.7 , but in this case 0.3 was a better choice.

A good test for the correctness of the solution is the density at the stagnation point, which can be easily calculated by inserting $M_{\infty}=0.5$ and $\rho_{\infty}=1.0$ in

$$
\rho_{\mathrm{o}}=\rho_{\infty}\left(1+\frac{\gamma-1}{2} M_{\infty}^{2}\right)^{1 /(\gamma-1)}
$$

giving $1 \cdot 129726$. The value obtained numerically is $1 \cdot 1320$, which differs by less than 2 per cent from the analytical one.

\subsection{Viscous supersonic flow over a flat plate}

The supersonic flow over a plate (Carter's flat plate problem) develops different features that can appear when solving the complete Navier-Stokes equations, such as boundary layers and shocks and the interaction between them. The state law is that of an ideal gas.
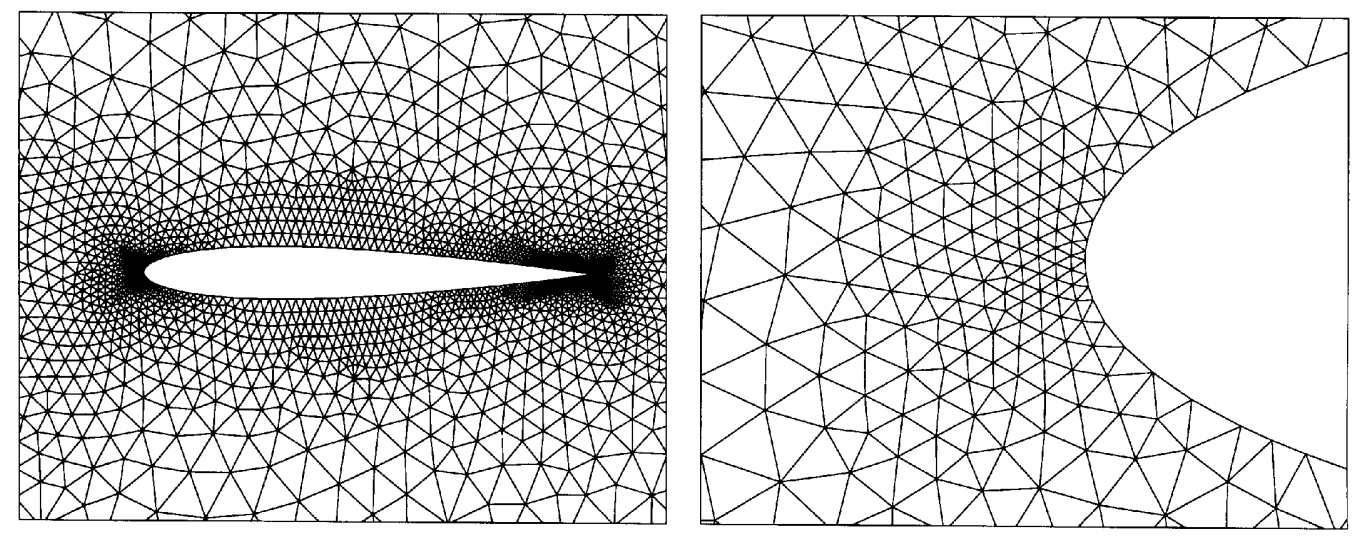

Figure 5. Flow over NACA 0012 profile: left, detail of mesh; right, stagnation point close-up 

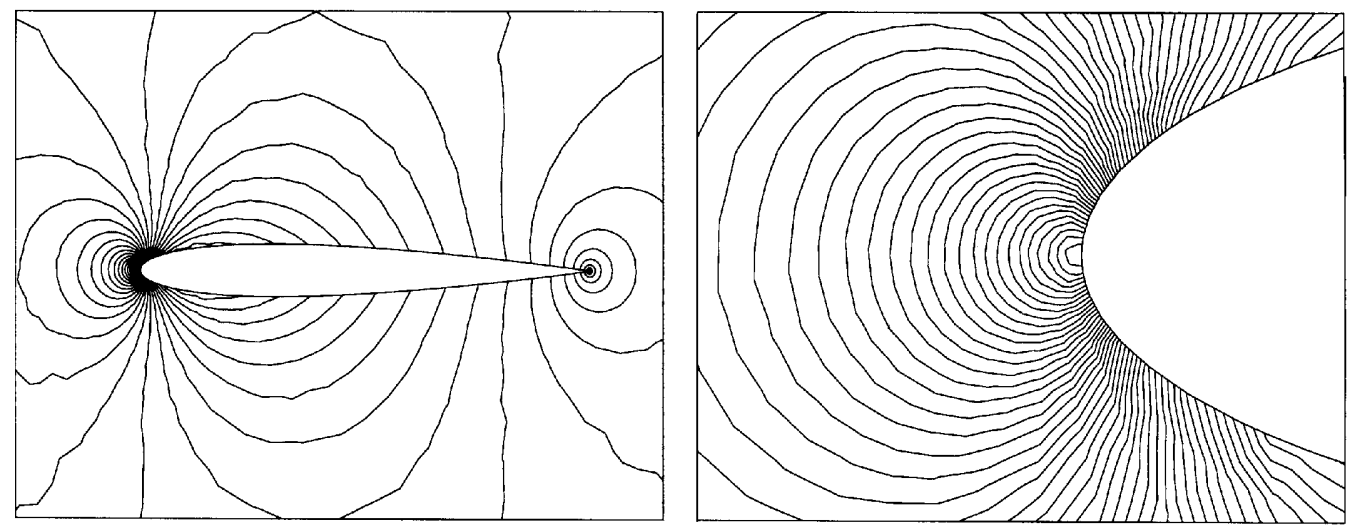

Figure 6. Flow over NACA 0012 profile: left, pressure isolines; right, stagnation point close-up

The Mach number at the inflow is $M_{\infty}=3 \cdot 0$. The viscosity $\mu$ depends on the temperature according to Sutherland's law:

$$
\mu=\frac{0 \cdot 0906 T^{1.5}}{T+0 \cdot 0001406} .
$$

The Prandtl number $\left(\operatorname{Pr}=\mu C_{\mathrm{p}} / k\right)$ is in this case $0 \cdot 72$, where $C_{\mathrm{p}}=\gamma C_{\mathrm{v}}$ is the specific heat at constant pressure, $C_{\mathrm{v}}=715$ and $\gamma=1.4$.

The domain is divided using a uniform mesh of $112 \times 64$ (7168) Q1 elements, corresponding to 7345 nodal points. If the co-ordinate origin is at the bottom left corner, the domain goes from $0 \cdot 0$ to 0.8 vertically and from 0.0 to 1.4 horizontally. The density, temperature and velocity are prescribed at the inflow, because this inlet is supersonic. The values prescribed at the inflow are 1.0 and $2 \cdot 8 \times 10^{-4}$ for the first two and $(1 \cdot 0,0 \cdot 0)$ for the horizontal and vertical components of the velocity. The no-slip condition is imposed on the floor of the plate, which starts at $x=0.25$.

The stagnation temperature is calculated according to

$$
T_{\text {stag }}=T_{\infty}\left(1+\frac{\gamma-1}{2} M_{\infty}^{2}\right)
$$

which is the prescription of this variable along the plate. No prescriptions are made at the outflow. This point must be remarked, because most of the outlet is subsonic, eventually requiring a prescription of the density. Nevertheless, the only prescribed node of the outflow is that of the bottom right corner which is considered belonging to the plate, with its boundary conditions on temperature and velocity.

Figures 7-9 show the results obtained for this example. Note the sharpness of the shock and the gradual change of the variables along the boundary layer. In Figures 8 and 9 a comparison with the original results of Carter (as appearing in Reference 20) is made, showing good agreement with them. These figures correspond to the profiles of some variables along a cut at $x=1 \cdot 25$. The density, pressure and temperature are normalized using their inflow values.

For the velocity, density and temperature the only slight difference is in the very maximum value at the shock. Carter's pressure profile is not shown because it presents some oscillations. In this problem a shock-capturing diffusion is artificially applied according to Reference 15 and Part I of this work. $^{3}$ It is activated for all the equations. The algorithm works equally well for both the strong and weak compression regions of the domain, the shock and the boundary layer respectively. 

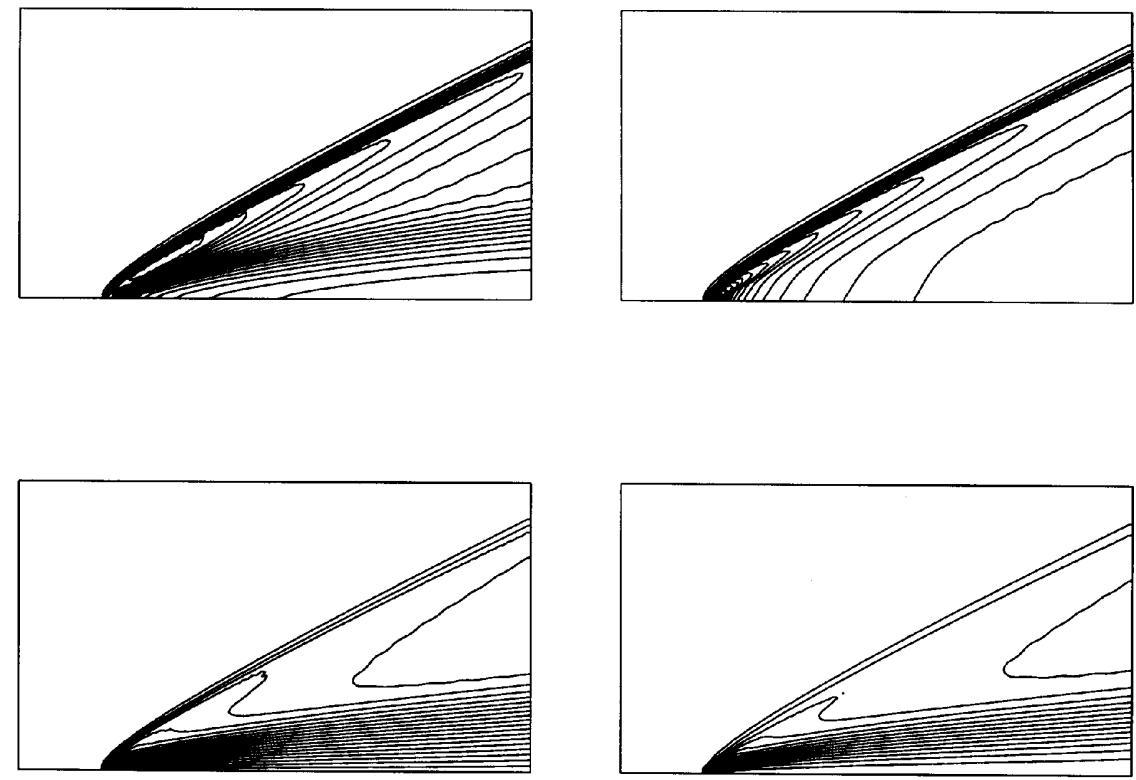

Figure 7. Flow over a plate: from top left, clockwise—density, pressure, temperature and Mach number level contours

\section{CONCLUSIONS}

In this paper we have discussed several aspects related to the semi-implicit version of the fractional step method presented in Reference 3. Concerning the boundary conditions, it has been shown that the fractional momentum can be computed also on the boundaries. This needs the evaluation of the boundary integral of the viscous stresses for the Navier-Stokes equations. By doing this, the boundary conditions for the pressure can be those resulting directly from the momentum equations, thus avoiding numerical boundary layers present in other fractional step methods for incompressible flow problems.
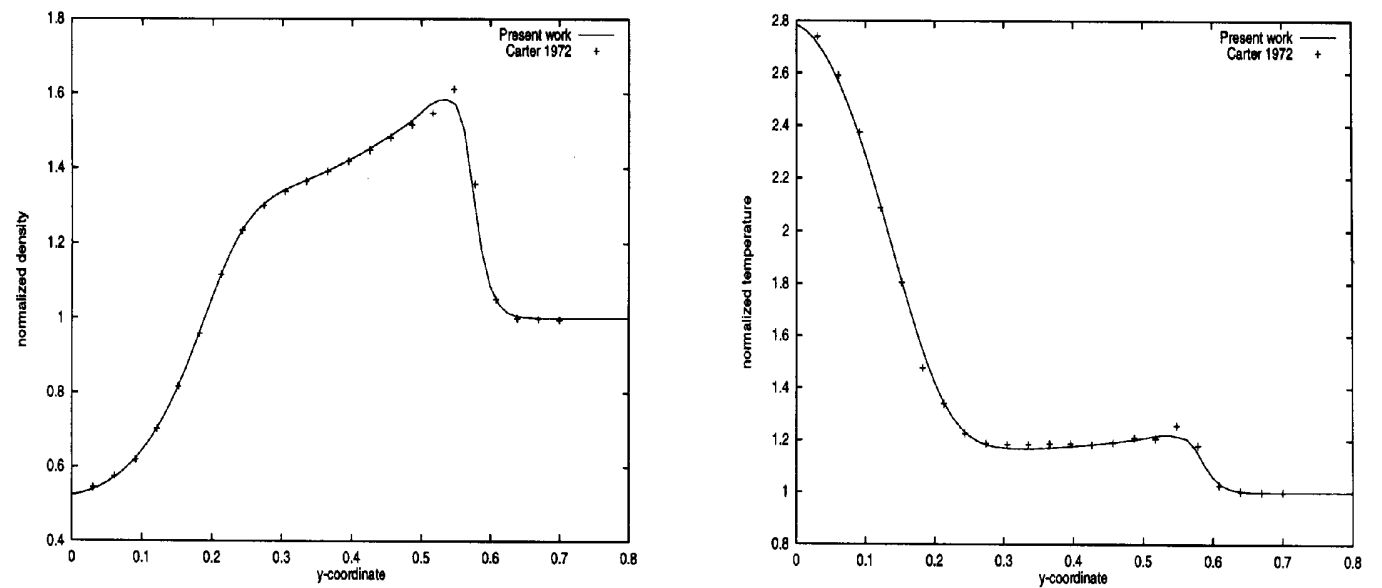

Figure 8. Flow over a plate: density and temperature (normalized using their inflow values) along a vertical cut at $x=1.25$ 

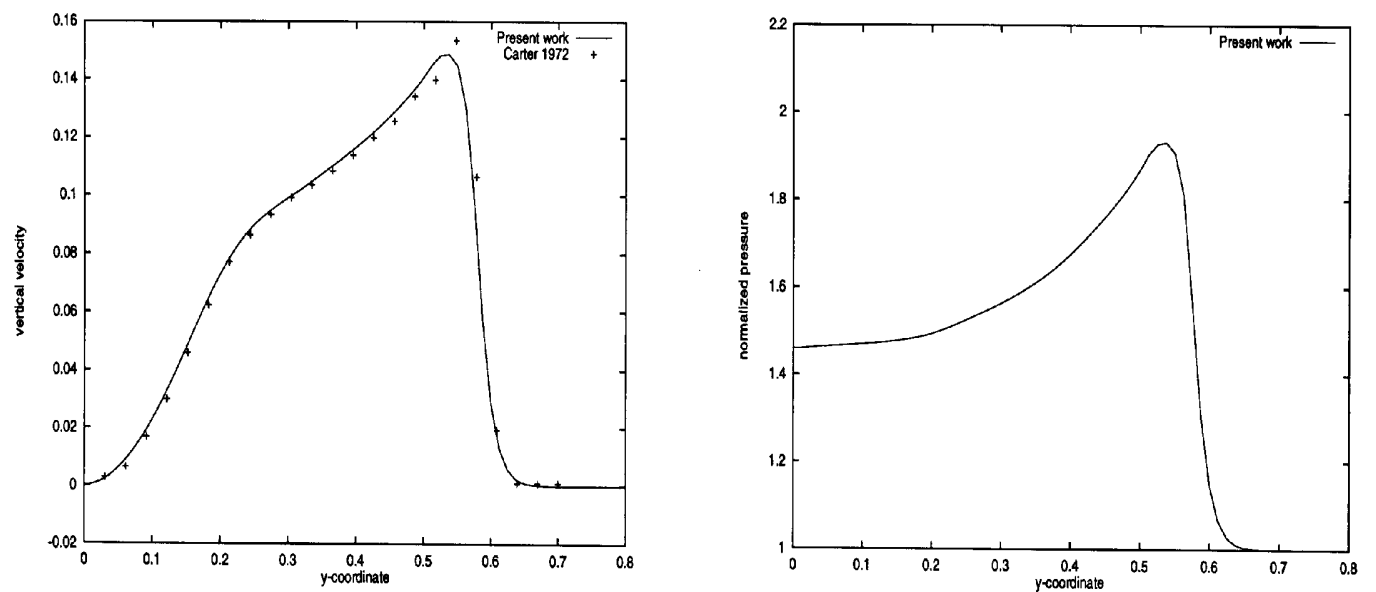

Figure 9. Flow over a plate: vertical component of velocity and normalized pressure (normalized using their inflow values) along a vertical cut at $x=1.25$

The semi-implicit form of the algorithm allows us to solve incompressible flow problems. We have provided an explanation for the fact that it is possible to use equal velocity-pressure interpolation with some fractional step methods. We have shown that they introduce a pressure stabilization term that is basically the difference between two discrete Laplacian operators computed in a different manner.

We have also discussed the solution of barotropic flows and flows of perfect gases. In this case one has to choose either the density or the pressure as the unknown for the continuity equation. We have shown that for all the flow types considered this equation has the same structure, even in the case of perfect gases, for which the RHS needs to be modified owing to the temperature variation. It is because of this that the algorithm proposed here may be considered general, especially using its semiimplicit form.

Numerical examples for incompressible flow, barotropic flow and compressible flow of a perfect gas have been presented (see Reference 18 for many more numerical examples), showing that the algorithm behaves very well for these three types of flow regimes.

\section{ACKNOWLEDGEMENT}

This research has been partially supported by NASA grant NAGW/2127, Ames Control Number 90-144.

\section{REFERENCES}

1. A. J. Chorin, 'Numerical solution of the Navier-Stokes equations', Math. Comput., 22, 745-762 (1968).

2. R. Temam, 'Sur l'approximation de la solution des équations de Navier-Stokes par la méthode des pas fractionaires (I)', Arch. Rat. Mech. Anal., 32, 135-153 (1969).

3. O. C. Zienkiewicz and R. Codina, 'A general algorithm for compressible and incompressible flow. Part I: The split, characteristic based scheme', Int. j. numer. methods fluids, 20, 869-885 (1995).

4. O. C. Zienkiewicz, K. Morgan, B. V. K. Satya Sai, R. Codina and M. Vázquez, 'A general algorithm for compressible and incompressible flow. Part II: Tests on the explicit form', Int. j. numer. methods fluids, 20, 887-913 (1995).

5. O. C. Zienkiewicz and J. Wu, 'A general explicit or semi-explicit algorithm for compressible or incompressible flows', Int. j. numer. methods eng., 35, 457-479 (1992). 
6. O. C. Zienkiewicz, B. V. K. Satya Sai, K. Morgan and R. Codina, 'Split, characteristic based semi-implicit algorithm for laminar/turbulent incompressible flows', Int. j. numer. methods fluids, 23, 787-809 (1996).

7. R. Codina and J. Blasco, 'A finite element formulation for the Stokes problem allowing equal velocity-pressure interpolation', Comput. Methods Appl. Mech. Eng., 143, 373-391 (1997).

8. M. O. Bristeau, R. Glowinsky, L. Dutto and J. Periaux, 'Compressible viscous flow calculations using compatible finite element approximations', Int. j. numer. methods fluids, 11, 719-749 (1990).

9. A. Soulaimani and M. Fortin, 'Finite element solution of compressible viscous flows using conservative variables', Comput. Methods Appl. Mech. Eng., 118, 319-350 (1994).

10. O. C. Zienkiewicz, J. Szmelter and J. Peraire, 'Compressible and incompressible flow; an algorithm for all seasons', Comput. Methods Appl. Mech. Eng., 78, 105-121 (1990).

11. O. Pironneau and J. Rappaz, 'Numerical analysis for compressible viscous isentropic stationary flows', Impact Comput. Sci. Eng., 1, 109-137 (1989).

12. F. Shakib, T. J. R. Hughes and Z. Johan, 'A new finite element formulation for computational fluid dynamics: X. The compressible Euler and Navier-Stokes equations', Comput. Methods Appl. Mech. Eng., 89, 141-219 (1991).

13. G. Hauke and T. J. R. Hughes, 'A unified approach to compressible and incompressible flows', Comput. Methods Appl. Mech. Eng., 113, 389-395 (1994).

14. T. C. Papanastasiou, N. Malamataris and K. Ellwood, 'A new outflow boundary condition', Int. j. numer. methods fluids, 14, 587- 608 (1992).

15. R. Codina, 'A discontinuity-capturing crosswind-dissipation for the finite element solution of the convection-diffusion equation', Comput. Methods Appl. Mech. Eng., 110, 325-342 (1993).

16. G. E. Schneider, G. D. Raithby and M. M. Yovanovich, 'Finite element analysis of incompressible fluid flow incorporating equal order pressure and velocity interpolation', in C. Taylor, K. Morgan and C. A. Brebbia (eds), Numerical Methods in Laminar and Turbulent Flow, Pentech, Plymouth, 1978.

17. M. Kawahara and K. Ohmiya, 'Finite element analysis of density flow using the velocity correction method', Int. $j$. numer. methods fluids, 5, 981-993 (1985).

18. M. Vázquez, R. Codina and O. C. Zienkiewicz, 'A fractional step method for the solution of Navier-Stokes equations', CIMNE Rep. 103, 1996.

19. U. Ghia, K. N. Ghia and C. T. Shin, 'High-Re solutions for incompressible flow using the Navier-Stokes equations and a multi-grid method', J. Comput. Phys., 48, 387-441 (1982).

20. L. Demkowicz, J. T. Oden and W. Rachowicz, 'A new finite element method for solving compressible Navier-Stokes equations based on an operator splitting method and $h-p$ adaptivity', Comput. Methods Appl. Mech. Eng., 84, 275-326 (1990). 